\title{
Synthesis and Optical Properties of
}

Fluorescent Switchable Silica

\section{Nanoparticles Covered with Copolymers} Consisting of Diarylethene and Fluorene Derivatives

\section{Katsuya Shimizu, Seiya Kobatake}

\begin{tabular}{|c|l|}
\hline Citation & ChemistrySelect. 2(20); $5445-5452$ \\
\hline Issue Date & $2017-07-11$ \\
\hline Type & Journal Article \\
\hline Textversion & author \\
\hline \multirow{2}{*}{ Rights } & $\begin{array}{l}\text { This is the peer-reviewed version of the following article: Shimizu Katsuya and } \\
\text { Kobatake Seiya. (2017), Synthesis and Optical Properties of Fluorescent } \\
\text { Switchable Silica Nanoparticles Covered with Copolymers Consisting of } \\
\text { Diarylethene and Fluorene Derivatives.ChemistrySelect.,2: 5445-5452, which has } \\
\text { been published in final form at https://doi.org/10.1002/slct.201700698. This article } \\
\text { may be used for non-commercial purposes in accordance with Wiley-VCH Terms } \\
\text { and Conditions for Self-Archiving. }\end{array}$ \\
\hline DOI & $\begin{array}{l}\text { This is the accepted manuscript version. Please cite only the published version. } \\
\text { 引用の際には出版社版をご確認ご利用ください。 }\end{array}$ \\
\hline
\end{tabular}

Self-Archiving by Author(s)

Placed on: Osaka City University

SHIMIZU, K., \& KOBATAKE, S. (2017). Synthesis and Optical Properties of Fluorescent Switchable Silica Nanoparticles Covered with Copolymers Consisting of Diarylethene and Fluorene Derivatives. ChemistrySelect. 2, 5445-5452. 


\section{WILEY-VCH}

\section{Synthesis and Optical Properties of Fluorescent Switchable Silica Nanoparticles Covered with Copolymers Consisting of Diarylethene and Fluorene Derivatives}

Katsuya Shimizu and Seiya Kobatake*

K. Shimizu, Prof. S. Kobatake

Department of Applied Chemistry, Graduate School of Engineering, Osaka City University, 3-3-138 Sugimoto, Sumiyoshi-ku, Osaka 558-8585, Japan

E-mail: kobatake@a-chem.eng.osaka-cu.ac.jp

\section{Abstract}

Silica nanoparticles covered with random copolymers consisting of diarylethene monomer (DE) and fluorene monomer (FL) (SNP-poly(DE-co-FL)) were designed and synthesized by reversible addition-fragmentation chain transfer (RAFT) copolymerization of DE and FL initiated from a RAFT agent immobilized on the surface of silica nanoparticle. SNP-poly(DE$c o-\mathrm{FL})$ and silica-free polymer (poly(DE-co-FL)), which are soluble in tetrahydrofuran, underwent reversible photoisomerization of DE moieties upon alternating irradiation with ultraviolet and visible light. They exhibited fluorescence ON/OFF switching between fluorescence $\mathrm{ON}$ state of FL in the open-ring form of DE and fluorescence OFF state by an efficient Förster/fluorescence resonance energy transfer from the excited FL not only in the same polymer chain but also in the surrounding polymer chains to the closed-ring form of DE. Diarylethene-fluorene dyad (DE-FL) was synthesized to compare with fluorescence ON/OFF switching efficiencies of SNP-poly(DE-co-FL) and poly(DE-co-FL). The closed-ring isomer of DE in DE-FL, poly(DE-co-FL), and SNP-poly(DE-co-FL) was found to quench fluorescence of $0.78,6.3$, and 15.6 FL moieties, respectively. Thus, fluorescence switching efficiency of SNP-poly(DE-co-FL) was 2.5 times higher than poly(DE-co-FL) and 20 times higher than DE-FL. Furthermore, the closed-ring isomer of DE in SNP-poly(DE-co-FL) was found to quench 6.3 FL moieties in the same polymer chain and 9.3 FL moieties in four polymer chains in the neighborhood. 


\section{WILEY-VCH}

\section{Introduction}

Photochromic compounds exhibit reversible transformation between two isomers upon irradiation at an appropriate wavelength of light. ${ }^{[1,2]}$ Photochromic compounds have drawn much attention because of their potential applications to switching devices in the optoelectronics field. ${ }^{[3-6]}$ Furthermore, fluorescence switching using photochromic molecules is expected to be useful for optical memory, super resolution microscopy, metal ion detection, and thermosensor in living cells because they can change the fluorescence intensities and colors by response to external stimuli, such as light, temperature, $\mathrm{pH}$, and metal ions. ${ }^{[7,8]}$ Among photochromic compounds, diarylethenes are typical P-type photochromic compounds and undergo reversible photoisomerization upon alternating irradiation with ultraviolet (UV) and visible light. The interconversion between two states accompanies the changes in physical properties, such as absorption spectra, fluorescence, refractive indices, dielectric constants, and oxidation-reduction potentials. ${ }^{[9]}$

Fluorescence switching enables us to visualize phenomena in micron area, but rapid response to external stimuli and large changes in fluorescence intensities and colors by the stimuli are required for applications in the bioimaging field. In particular, ON-OFF switching of fluorescence by light has been investigated in these days. ${ }^{[9,10]}$ In the case of diarylethene derivatives linked to fluorophore, fluorescence is emitted from fluorophore when diarylethene exists in only open-ring form. On the other hand, when diarylethene transforms to the closedring form, it is not emitted because of Förster/fluorescence resonance energy transfer (FRET) from fluorophore to the closed-ring form. ${ }^{[11]}$ The change in fluorescence intensity strongly depends on photoisomerization conversion, and the fluorescence intensity decreases in approximately proportional to increasing photocyclization conversion in a dyad system of fluorophore and diarylethene photochromic dyes. For instance, when the photocyclization conversion of diarylethene is $70 \%, 30 \%$ of fluorescence remains even in high efficiency because the diarylethene closed-ring isomer can quench the fluorescence of only 


\section{WILEY-VCH}

intramolecular fluorophore. If fluorescence intensity can be efficiently switched in low conversion in photochromic reactions, high fluorescence switching speed and high fatigueresistance can be expected. Moreover, FRET efficiency is generally inversely proportional to $r^{6}\left(r\right.$ : intermolecular distance between donor and acceptor moieties).$^{[12,13]}$ To date, various types of fluorescence switchable diarylethenes with high efficiency of fluorescence switching, high fluorescence quantum yield $\left(\Phi_{\mathrm{f}}\right)$, and high ON/OFF contrast have been developed. ${ }^{[10,11,14-19]}$ In particular, Fölling and co-workers reported that silica nanoparticle incorporated with diarylethene-rhodamine dyad exhibited high fluorescence quenching even in low photocyclization conversion. ${ }^{[19]}$ In such systems, $75 \%$ of fluorescence was quenched even in $20 \%$ photocyclization conversion. However, design and development of novel fluorescent switchable molecule with high-speed switching capability are desired.

Here, we report design and fabrication of silica nanoparticles covered with random copolymers consisting of diarylethene and fluorene derivatives for an efficient fluorescence ON/OFF switching, as shown in Figure 1. Silica surface-initiated polymerization was conducted by reversible addition-fragmentation chain transfer (RAFT) copolymerization of diarylethene and fluorene monomers (DE and FL, respectively) using silica nanoparticle covered with a RAFT agent. ${ }^{[20-22]}$ The fluorescence switching behavior of silica nanoparticle covered with fluorescent switchable polymers (SNP-poly(DE-co-FL)) is discussed in this paper. 


\section{WILEY-VCH}
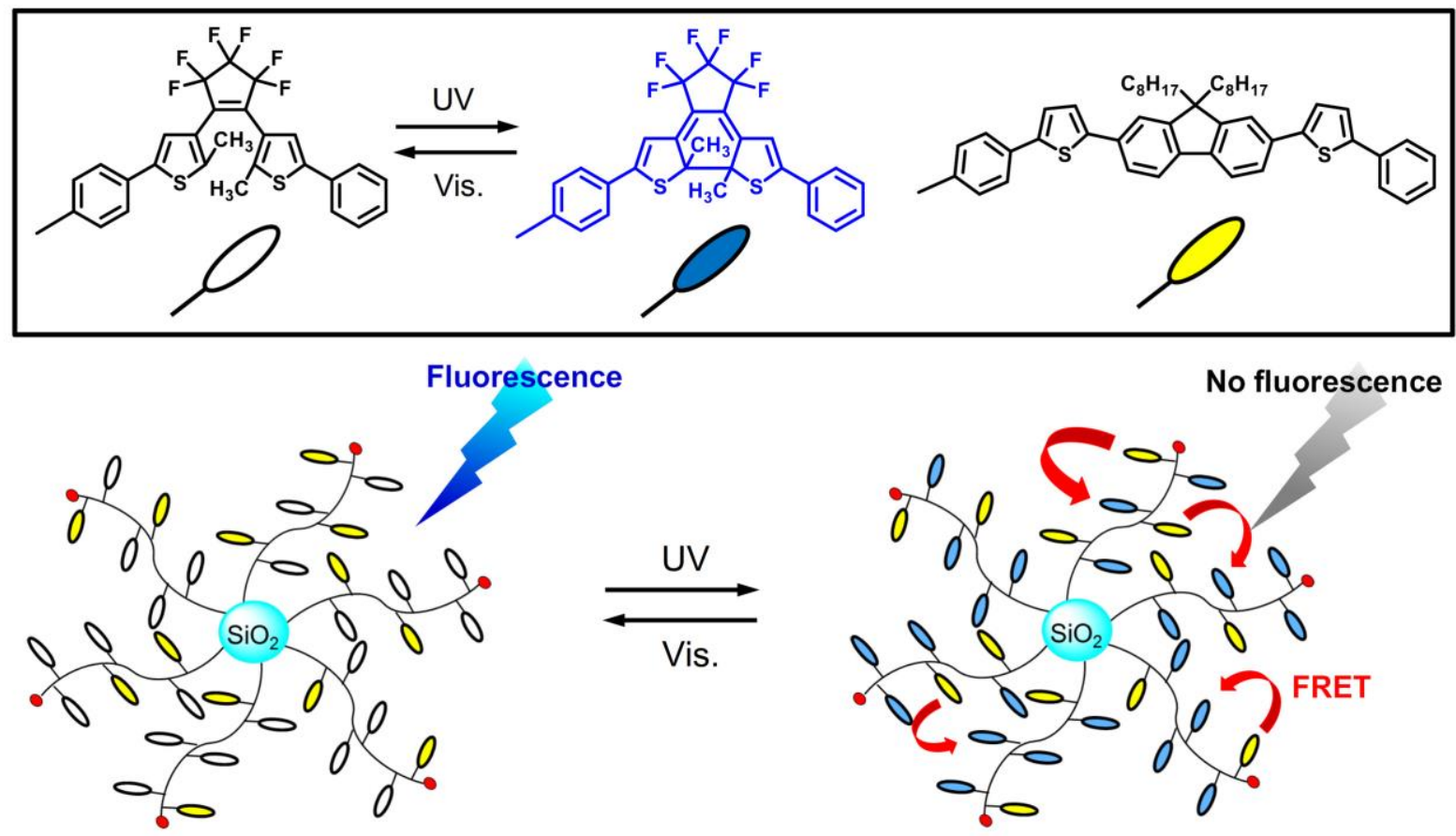

Figure 1. Silica nanoparticle covered with copolymers consisting of diarylethene and fluorene derivatives used in this work.

\section{Results and Discussion}

\subsection{Synthesis and Characterization of SNP-poly(DE-co-FL)}

Silica nanoparticle (SNP) was synthesized by the Stöber method that can produce a monodispersed SNP with small size distribution. ${ }^{[23]}$ Figure 2 shows SEM image of SNP fabricated in this work. SNP was independently dispersed with the size of ca. $100 \mathrm{~nm}$. The size of SNP was also estimated by DLS. The average particle diameter $(D)$ and the standard deviation $(\sigma)$ of SNP were determined to be 109 and $8.9 \mathrm{~nm}$, respectively. This indicates that SNP has narrow particle size distribution $(\sigma D=8.2 \%)$. The resulting SNP was treated with hydrochloric acid and washed with distilled water, followed by refluxing a tetrahydrofuran (THF) solution of a RAFT agent with a methoxydimethylsilyl group. ${ }^{[20]}$ The siloxane bond was formed by the reaction of the methoxysilyl group and the silanol group on the SNP surface. The resulting SNP covered with the RAFT agent (SNP-RAFT) has a pale red color 


\section{WILEY-VCH}

originated from dithiobenzoate group. This means that the RAFT agent is successfully immobilized onto SNP.
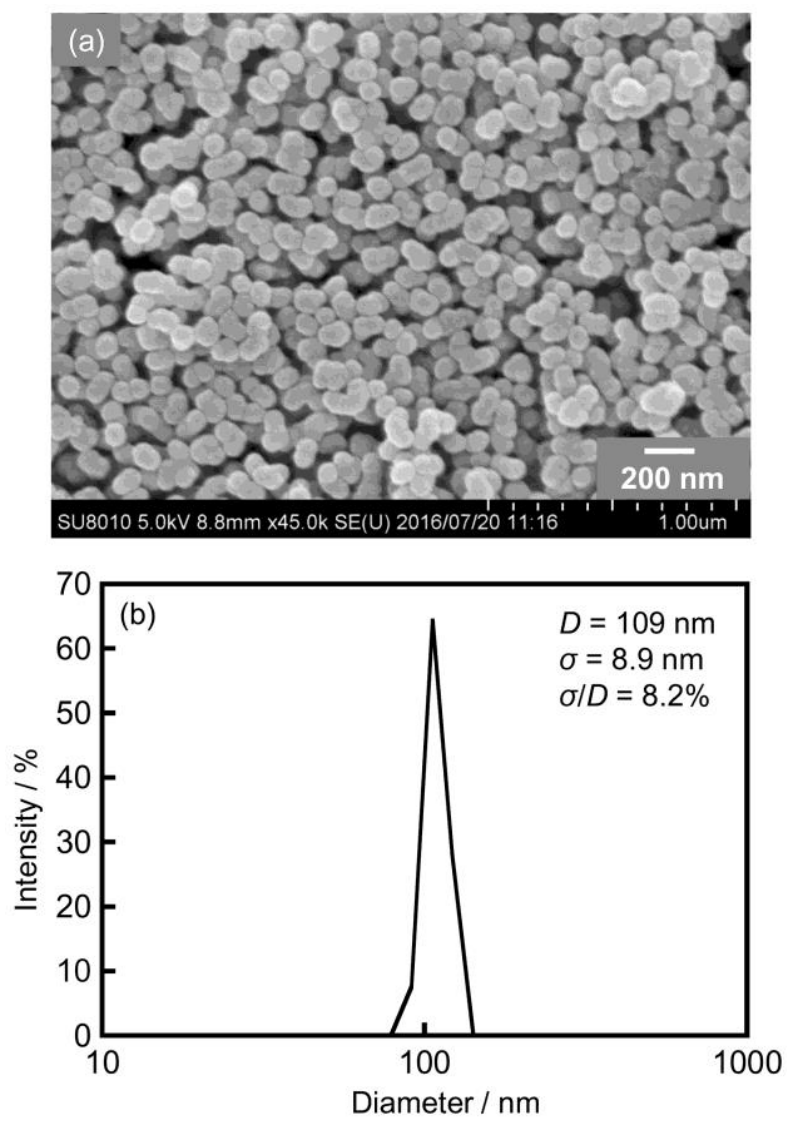

Figure 2. (a) SEM image and (b) DLS profile of SNP.

RAFT copolymerization of DE and FL using SNP-RAFT as the RAFT agent was conducted at $100{ }^{\circ} \mathrm{C}$. RAFT polymerization using SNP-RAFT without a silica-free RAFT agent does not control $M_{\mathrm{n}}$ and $M_{\mathrm{w}} / M_{\mathrm{n}}$ of the grafting polymers onto SNP. ${ }^{[24]}$ Therefore, FRAFT as the silica-free RAFT agent was added to the polymerization mixture (Scheme 1). Table 1 shows the polymerization conditions and results. SNP-poly(FL), SNP-poly(DE), and SNP-poly(DE-co-FL) covered with polymers or copolymers were successfully synthesized. The copolymer composition was determined by ${ }^{1} \mathrm{H}$ NMR spectroscopic analysis of methyl protons for $\mathrm{DE}$ and methylene protons bound to 9-carbon of fluorene for FL in the copolymers (Figure S1 in the Supporting Information). It was reported that the molecular 


\section{WILEY-VCH}

weight of polymer chain on the surface of SNP is equal to that of silica-free polymer. ${ }^{[25]} M_{\mathrm{n}}$ and $M_{\mathrm{w}} / M_{\mathrm{n}}$ of silica-free polymer instead to those of polymer chain on the surface of SNP are summarized in Table 1. SNP-poly(DE-co-FL) with $M_{\mathrm{n}}$ of 10700 was successfully fabricated. Figure 3 shows SEM image and DLS profile of SNP-poly(DE-co-FL). SNP-poly(DE-co-FL) has the average diameter of $177 \mathrm{~nm}$ with the standard deviation of $12.8 \mathrm{~nm}$. The particle size increased in $68 \mathrm{~nm}$ larger by the coverage of poly(DE-co-FL). This indicates that SNP was successfully coveraged by poly(DE-co-FL).

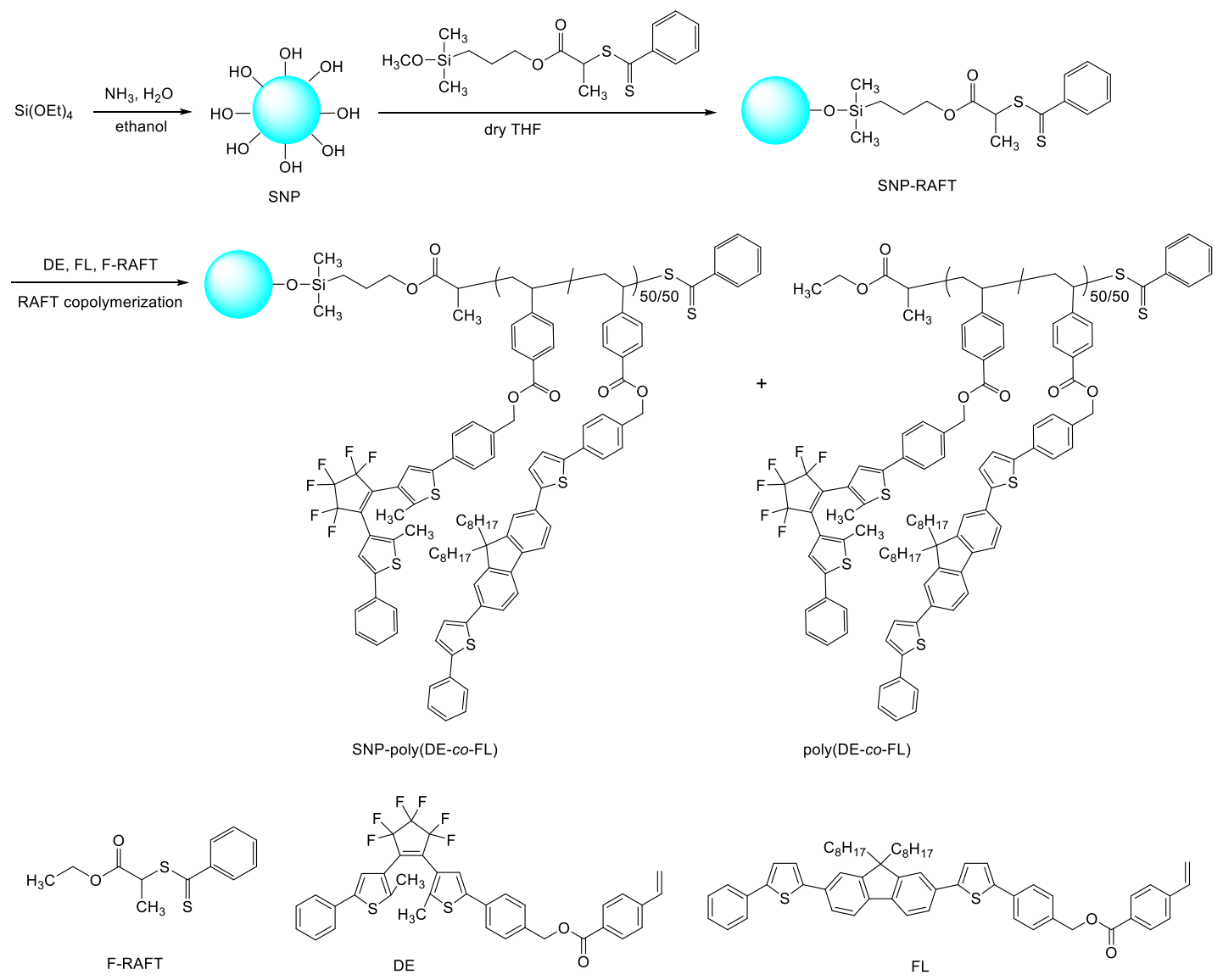

Scheme 1. Synthetic routes of silica nanoparticle covered with copolymers consisting of DE and FL. 


\section{WILEY-VCH}

Table 1. RAFT polymerization or copolymerization of DE and FL in toluene at $100{ }^{\circ} \mathrm{C}^{\text {[a] }}$

\begin{tabular}{cccccc}
\hline $\begin{array}{c}{[\mathrm{DE}]} \\
{\left[\mathrm{mol} \mathrm{L}^{-1}\right]}\end{array}$ & $\begin{array}{c}{[\mathrm{FL}]} \\
{\left[\mathrm{mol} \mathrm{L}^{-1}\right]}\end{array}$ & $\begin{array}{c}\text { Time } \\
{[\mathrm{h}]}\end{array}$ & $\begin{array}{c}M_{\mathrm{n}} \\
(\mathrm{GPC})\end{array}$ & $\begin{array}{c}M_{\mathrm{w}} / M_{\mathrm{n}} \\
(\mathrm{GPC})\end{array}$ & $\begin{array}{c}\text { DE : FL } \\
(\text { molar ratio) }\end{array}$ \\
\hline 0 & 0.45 & 45 & 33600 & 1.11 & $0: 100$ \\
0.20 & 0.20 & 40 & 10700 & 1.20 & $50: 50^{[\mathrm{b}]}$ \\
0.45 & 0 & 45 & 16200 & 1.19 & $100: 0$ \\
\hline
\end{tabular}

[a] RAFT agent: [SNP-RAFT] $=50 \mathrm{mg} \mathrm{mL}^{-1},[\mathrm{~F}-\mathrm{RAFT}]=5.0 \times 10^{-3} \mathrm{~mol} \mathrm{~L}^{-1}$, Initiator: $[$ ATMP] $=$ $2.5 \times 10^{-4} \mathrm{~mol} \mathrm{~L}^{-1}$. [b] Molar fraction in copolymer.
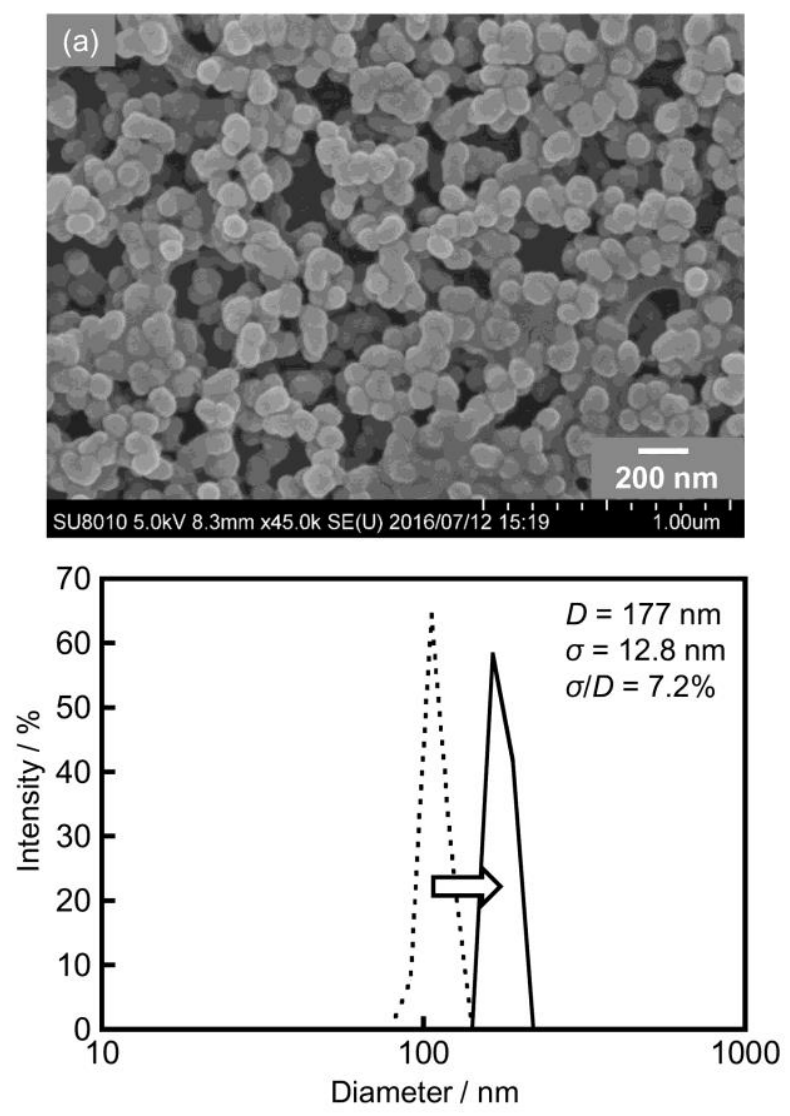

Figure 3. (a) SEM image and (b) DLS profile of SNP-poly(DE-co-FL).

The coverage density of polymer chains on the surface of SNP was estimated as follows. The ratio of weight of polymer chains on the surface of SNP-poly(DE-co-FL) ( $\left.W_{\text {polymer }}\right)$ and weight of the SNP core in SNP-poly(DE-co-FL) ( $\left.W_{\mathrm{SNP}}\right)$ can be determined from thermogravimetry (TG) measurement, as shown in Figure 4. The number of polymer chains 


\section{WILEY-VCH}

on the surface of single SNP-poly(DE-co-FL) $\left(N_{\text {polymer }}\right)$ can be determined by Equation (1) using Avogadro's number $\left(N_{\mathrm{A}}\right)$ :

$$
\begin{aligned}
N_{\text {polymer }} & \left.=\frac{W_{\text {polymer }}}{W_{\mathrm{SNP}}} \times(\text { weight of SNP core in single SNP-poly(DE-co-FL })\right) \times \frac{N_{\mathrm{A}}}{M_{\mathrm{n}}} \\
& =\frac{W_{\text {polymer }}}{W_{\mathrm{SNP}}} \times \frac{4}{3} \pi\left(\frac{D}{2}\right)^{3} \rho \times \frac{N_{\mathrm{A}}}{M_{\mathrm{n}}}
\end{aligned}
$$

where $D$ and $\rho$ are diameter and density of SNP core in SNP-poly(DE-co-FL), respectively. The $D$ and $\rho$ values were used to be $109 \mathrm{~nm}$ and $2.0 \mathrm{~g} \mathrm{~cm}^{-3}$. [26]
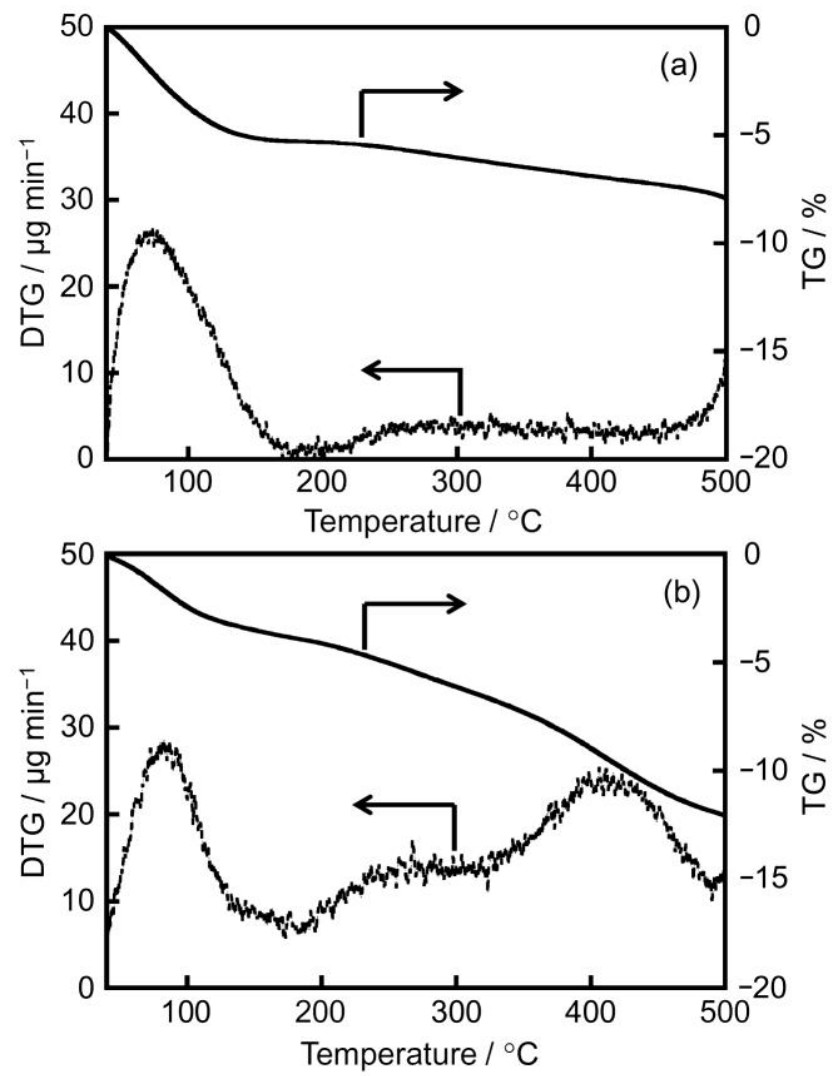

Figure 4. TG curve ( $—$ ) and DTG curve ( - - ) of (a) SNP and (b) SNP-poly(DE-co-FL).

The coverage density $(d)$ of the polymer chains on the surface of SNP in SNP-poly(DEco-FL) can be determined by Equation (2): 


$$
\begin{aligned}
d & =\frac{N_{\text {polymer }}}{(\text { Surface area on SNP })} \\
& =\frac{N_{\text {polymer }}}{4 \pi(D / 2)^{2}} \\
& =\frac{W_{\text {polymer }}}{W_{\text {SNP }}} \times \frac{D \rho}{6} \times \frac{N_{\mathrm{A}}}{M_{\mathrm{n}}}
\end{aligned}
$$

$W_{\text {polymer }} / W_{\text {SNP }}$ can be determined from weight loss by decomposition of the polymers in SNP-poly(DE-co-FL) by TG measurement in the rate of temperature rise of $10{ }^{\circ} \mathrm{C} \mathrm{min}^{-1}$ under the temperature range of $40-500{ }^{\circ} \mathrm{C}$. In contrast, the weight of silica nanoparticle core can be determined by subtracting weight of decomposition from total weight. The weight loss in entire temperature region is derived from decomposition of polymers and dehydration of unreacted silanol groups by heating. Therefore, the accurate polymer fraction can be determined by comparing TG measurements of SNP and SNP-poly(DE-co-FL). First, the weight loss of SNP below $170{ }^{\circ} \mathrm{C}$ is due to water absorbed on the surface of SNP and was determined to be $5.3 \mathrm{wt} \%$. The weight loss at $500{ }^{\circ} \mathrm{C}$ was $7.9 \mathrm{wt} \%$. The subtraction of the weight loss at 170 and $500{ }^{\circ} \mathrm{C}(2.6 \mathrm{wt} \%)$ is due to dehydration of unreacted silanol groups. Next, TG measurement of SNP-poly(DE-co-FL) was conducted. The weight loss of SNPpoly(DE-co-FL) below $170{ }^{\circ} \mathrm{C}$ was $3.8 \mathrm{wt} \%$. The weight loss at $500{ }^{\circ} \mathrm{C}$ was $12.1 \mathrm{wt} \%$. From these results, $W_{\text {polymer }} / W_{\text {SNP }}$ was calculated to be $6.5 / 87.9((12.1-3.8 \times 7.9 / 5.3) /(100-12.1)=$ 6.5/87.9). Therefore, the coverage density was estimated to be $d=0.15$ chains $\mathrm{nm}^{-2}$. In other words, copolymers are formed at intervals of ca. $2.6 \mathrm{~nm}$ (Figure S2 in the Supporting Information). This means that 5600 polymer chains covered single SNP. These results indicate that copolymers of DE and FL were formed densely on the surface of SNP. ${ }^{[27]}$

\subsection{Optical Properties of DE-FL}

DE-FL underwent reversible photochromic reactions upon irradiation with UV and visible light, as shown in Figure 5a. Upon irradiation with $313 \mathrm{~nm}$ light, new absorption band 


\section{WILEY-VCH}

appeared at 450-700 $\mathrm{nm}$ in THF, and the absorption band disappeared upon irradiation with visible light. Figure $5 \mathrm{~b}$ shows fluorescence spectral changes of DE-FL in THF upon alternating irradiation with UV and visible light. DE-FL exhibited strong fluorescence derived from the FL moiety. Table 2 summarizes the results. Fluorescence maximum wavelength was observed at $475 \mathrm{~nm}$. Fluorescence quantum yield of DE-FL was determined to be $\Phi_{\text {s,open }}=$ 0.70 in the open-ring form of DE. Because absorption spectrum of the closed-ring form (DEc) is overlapped with fluorescence spectrum of FL, as shown in Figure S3 in the Supporting Information, the fluorescence intensity decreased due to an excited state energy transfer from FL to DE-c. However, fluorescence of DE-FL was not completely quenched even at the photostationary state (PSS). In our system, the spectral overlap between fluorescence of FL and absorption of DE-c is not so large. Therefore, although the photocyclization conversion at PSS reaches to $97 \%$, the fluorescence quenching efficiency from FL to DE-c is as small as 0.78. Here, we define fluorescence modulation $\left(1-F / F_{0}\right)$ as the initial fluorescence intensity relative to the fluorescence intensity at a photocyclization conversion upon irradiation with $313 \mathrm{~nm}$ light. The fluorescence modulation for DE-FL at PSS was determined to be $78 \%$. This means that $78 \%$ of fluorescence intensity at the initial state was quenched at PSS. Figure 6a shows a normalized fluorescence intensity relative to the photocyclization conversion of DEFL in THF. The fluorescence intensity decreased linearly with increasing photocyclization conversion. 


\section{WILEY-VCH}

Table 2. Optical properties of DE-FL, poly(DE-co-FL), and SNP-poly(DE-co-FL).

\begin{tabular}{|c|c|c|c|c|c|c|}
\hline & $\begin{array}{c}\lambda_{\mathrm{abs}} \\
{[\mathrm{nm}]}\end{array}$ & $\begin{array}{c}\lambda_{\text {flu }} \\
{[\mathrm{nm}]}\end{array}$ & $\begin{array}{c}\text { Conversion } \\
{[\%]^{[\mathrm{a}]}}\end{array}$ & $\Phi_{\mathrm{f}, \mathrm{open}}$ & $\begin{array}{c}\left(1-F / F_{0}\right) \times 100 \\
{[\%]^{[\mathrm{b}]}}\end{array}$ & $\begin{array}{c}R_{0} \\
{[\mathrm{~nm}]}\end{array}$ \\
\hline DE-FL & $394(\mathrm{FL}), 592(\mathrm{DE})$ & 475 & 97 & 0.70 & 78 & - \\
\hline $\operatorname{poly}(\mathrm{FL})$ & $389(\mathrm{FL})$ & 463 & - & 0.35 & - & - \\
\hline poly(DE-co-FL) & 390 (FL), 592 (DE) & 434 & 94 & 0.35 & 98 & 3.80 \\
\hline poly(DE) & $592(\mathrm{DE})$ & - & $97^{[\mathrm{c}]}$ & - & - & - \\
\hline SNP-poly(FL) & $389(\mathrm{FL})$ & 461 & - & 0.13 & - & - \\
\hline SNP-poly(DE-co-FL) & 394 (FL), 592 (DE) & 463 & 76 & 0.13 & 96 & 3.22 \\
\hline SNP-poly(DE) & $589(\mathrm{DE})$ & - & $96^{[\mathrm{c}]}$ & - & - & - \\
\hline
\end{tabular}

[a] Conversion from the open-ring form to the closed-ring form upon irradiation with $313 \mathrm{~nm}$ light. [b] At PSS. [c] Ref. 20. 


\section{WILEY-VCH}
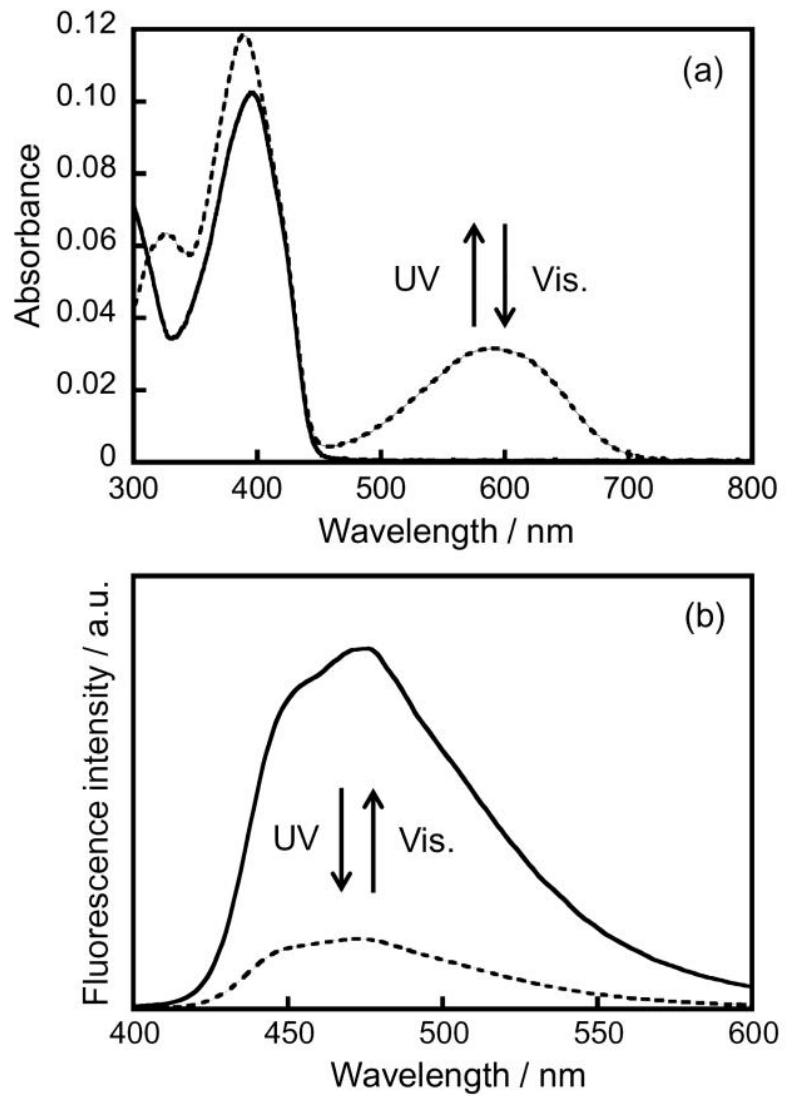

Figure 5. (a) Absorption and (b) fluorescence spectral changes of DE-FL in THF upon irradiation with $313 \mathrm{~nm}$. The fluorescence spectra were recorded upon excitation at $390 \mathrm{~nm}$.

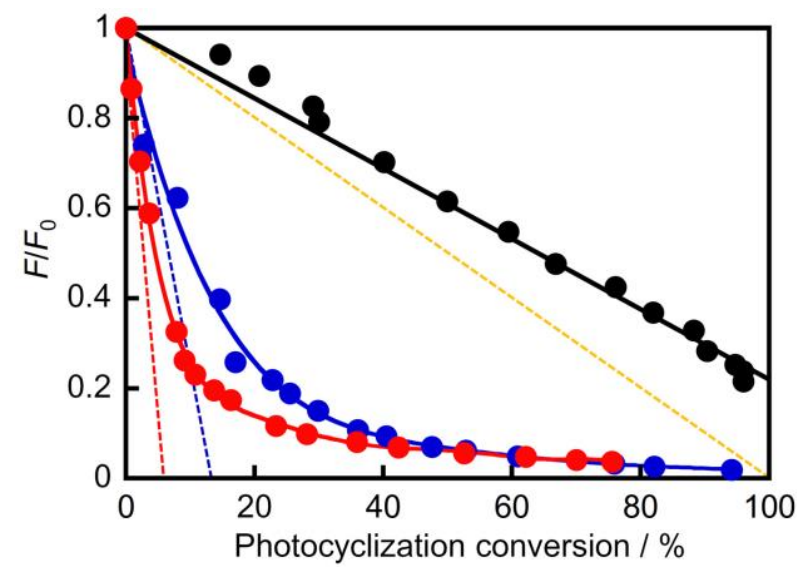

Figure 6. Normalized fluorescence intensity relative to the photocyclization conversion of DE-FL (black line), poly(DE-co-FL) (blue line), and SNP-poly(DE-co-FL) (red line) in THF upon irradiation with $313 \mathrm{~nm}$ light. Blue and red broken lines show the initial slope of the relationship between $F / F_{0}$ and photocyclization conversion for poly(DE-co-FL) and SNPpoly(DE-co-FL), respectively. 


\section{WILEY-VCH}

\subsection{Optical Properties of poly(DE-co-FL)}

Figure 7a shows absorption spectral changes of poly(DE-co-FL) in THF upon alternating irradiation with UV and visible light. Upon irradiation with $313 \mathrm{~nm}$ light, new absorption band appeared at 450-700 $\mathrm{nm}$, and the absorption band disappeared upon irradiation with visible light. Their spectral changes are similar to those of poly(DE) as shown in Figure S4a and Table S1 in the Supporting Information. The absorption spectrum of poly(FL) is shown in Figure S5a and Table S2 in the Supporting Information. The polymers underwent reversible photochromic reactions upon alternating irradiation with UV and visible light. Figure $7 \mathrm{~b}$ shows fluorescence spectral changes excited at $390 \mathrm{~nm}$ in THF upon alternating irradiation with UV and visible light. The fluorescence spectrum of FL in poly(DE-co-FL) is similar to that of poly(FL) as shown in Figure S5b in the Supporting Information. Table 2 also summarizes the results. Fluoresecence intensities for poly(FL) and poly(DE-co-FL) were weaker than that of DE-FL because of concentration quenching or energy migration of densely packed FL moieties. However, fluorescence intensity significantly decreased with increasing photocyclization conversion, as shown in Figure 6. This indicates that one DE-c molecule quenched fluorescence of some FL moieties. 

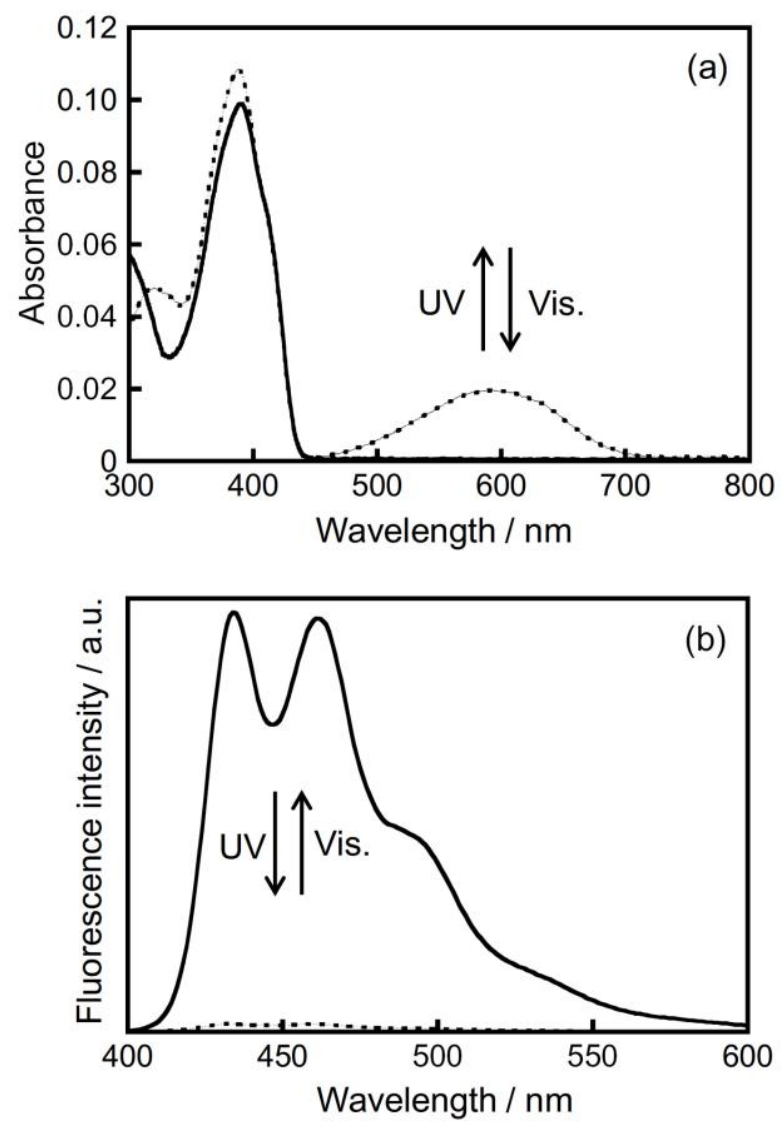

Figure 7. (a) Absorption and (b) fluorescence spectral changes of poly(DE-co-FL) in THF upon irradiation with $313 \mathrm{~nm}$. The fluorescence spectra were recorded upon excitation at 390 nm.

\subsection{Optical Properties of SNP-poly(DE-co-FL)}

SNP-poly(DE-co-FL) also exhibited photochromism as well as poly(DE-co-FL). Figure 8a shows absorption spectral changes of SNP-poly(DE-co-FL) in THF upon alternating irradiation with UV and visible light. The light scattering of the silica nanoparticles was observed in the measurement as an increase in the base line. No change of the light scattering was observed during the photochromic reactions. The similar behavior was also observed for SNP-poly(DE) as shown in Figure S4b and Table S1 in the Supporting Information. Figure 8b shows fluorescence spectral changes of SNP-poly(DE-co-FL) excited at $390 \mathrm{~nm}$ in THF upon alternating irradiation with UV and visible light. The fluorescence spectrum of FL is also similar to those of poly(FL) and SNP-poly(FL) as shown in Figure S5b,d and Table S2 in the Supporting Information. Table 2 also summarizes the results. Fluorescence of SNP-poly(DE- 


\section{WILEY-VCH}

co-FL) was weaker than that of poly(DE-co-FL). However, fluorescence intensity significantly decreased with increasing photocyclization conversion as it is for poly(DE-coFL), as shown in Figure 6. This indicates that one DE-c molecule quenched fluorescence of many FL moieties because one DE-c molecule is surrounded with much more FL moieties than poly(DE-co-FL). Silica nanoparticle reported by Fölling and co-workers exhibited that $75 \%$ of fluorescence was quenched in $20 \%$ photocyclization conversion. ${ }^{[19]}$ In our system, $88 \%$ of fluorescence can be quenched in the same conversion. We succeeded in preparing silica nanoparticle that exhibits efficient fluorescence switching.
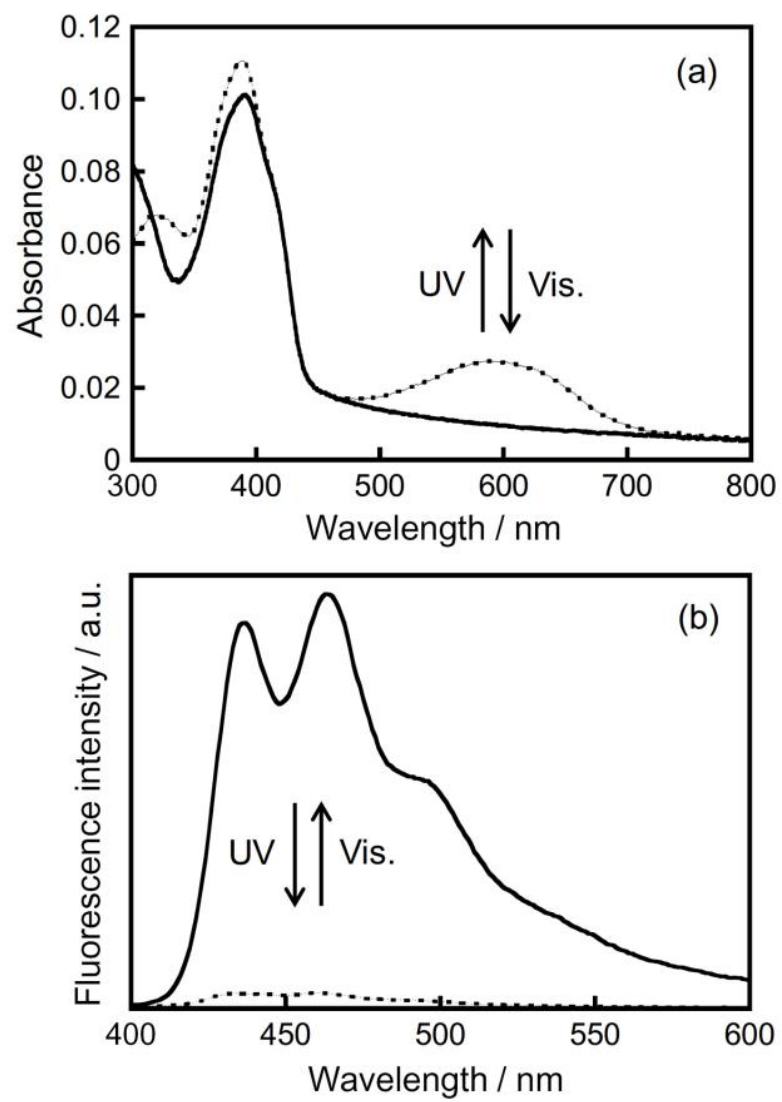

Figure 8. (a) Absorption and (b) fluorescence spectral changes of SNP-poly(DE-co-FL) in THF upon irradiation with $313 \mathrm{~nm}$. The fluorescence spectra were recorded upon excitation at $390 \mathrm{~nm}$.

\subsection{Fluorescence Switching Efficiency and Förster Distance}




\section{WILEY-VCH}

In order to evaluate the fluorescence switching efficiency quantitatively, we calculated the initial slope of the graph of normalized fluorescence intensity relative to the photocyclization conversion of DE-FL, poly(DE-co-FL), and SNP-poly(DE-co-FL). Here, the initial slope is equal to the number of fluorescence quenching per one DE-c molecule. For example, the orange-colored dotted line shows that one DE-c quenches fluorescence of one FL molecule because the fluorescence intensity become zero when photocyclization conversion is $100 \%$ (Figure 6). Then, initial slopes of approximate straight line in DE-FL and approximate curve line of poly(DE-co-FL) and SNP-poly(DE-co-FL) were calculated to be $0.78,6.3$, and 15.6, respectively. Therefore, it is indicated that one DE-c in DE-FL, poly(DEco-FL), and SNP-poly(DE-co-FL) can quench fluorescence from 0.78, 6.3, and 15.6 molecules of FL, respectively. Furthermore, Förster distances $\left(R_{0}\right)$ between DE-c and FL in poly(DE-co-FL) and SNP-poly(DE-co-FL) were calculated to be 3.80 and $3.22 \mathrm{~nm}$, respectively, from Equation (3), ${ }^{[12,13]}$ as shown in Table 2.

$$
R_{0}{ }^{6}=\frac{9 \Phi_{0}(\ln 10) \kappa^{2} J}{128 \pi^{5} n^{4} N_{\mathrm{A}}}
$$

where $\Phi_{0}$ is fluorescence quantum yield of donor, $\kappa^{2}$ is dipole orientation factor, $n$ is refractive index of solvent, $N_{\mathrm{A}}$ is Avogadro's number and $J$ is spectral overlapping described by Equation (4).

$$
J=\int f_{\mathrm{D}}(\lambda) \varepsilon_{\mathrm{A}}(\lambda) \lambda^{4} \mathrm{~d} \lambda
$$

where $f_{\mathrm{D}}(\lambda)$ is normalized fluorescence spectrum and $\varepsilon_{\mathrm{A}}(\lambda)$ is molar extinction coefficient of acceptor. These parameters are shown in Table S3 in the Supporting Information. One DE-c molecule in the polymer chain covered SNP can quench the fluorescence of FL inside a circle of $32.6 \mathrm{~nm}^{2}(=3.22 \mathrm{~nm} \times 3.22 \mathrm{~nm} \times 3.14)$ because the $R_{0}$ value for SNP-poly $(\mathrm{DE}-c o-\mathrm{FL})$ is $3.22 \mathrm{~nm}$. Here, taking $d\left(=0.15\right.$ chains $\left.\mathrm{nm}^{-2}\right)$ into consideration, the number of polymer chains inside the circle was estimated to be about 5 chains $\left(=32.6 \mathrm{~nm}^{2} \times 0.15\right.$ chains $\left.\mathrm{nm}^{-2}\right)$. In other words, one DE-c can quench fluorescence of FL in four polymer chains in the neighborhood. Accordingly, one DE-c in SNP-poly(DE-co-FL) is considered to quench 6.3 


\section{WILEY-VCH}

molecules of FL in the same polymer chain and 9.3 molecules of FL in four polymer chains in the neighborhood, as shown in Figure 9.

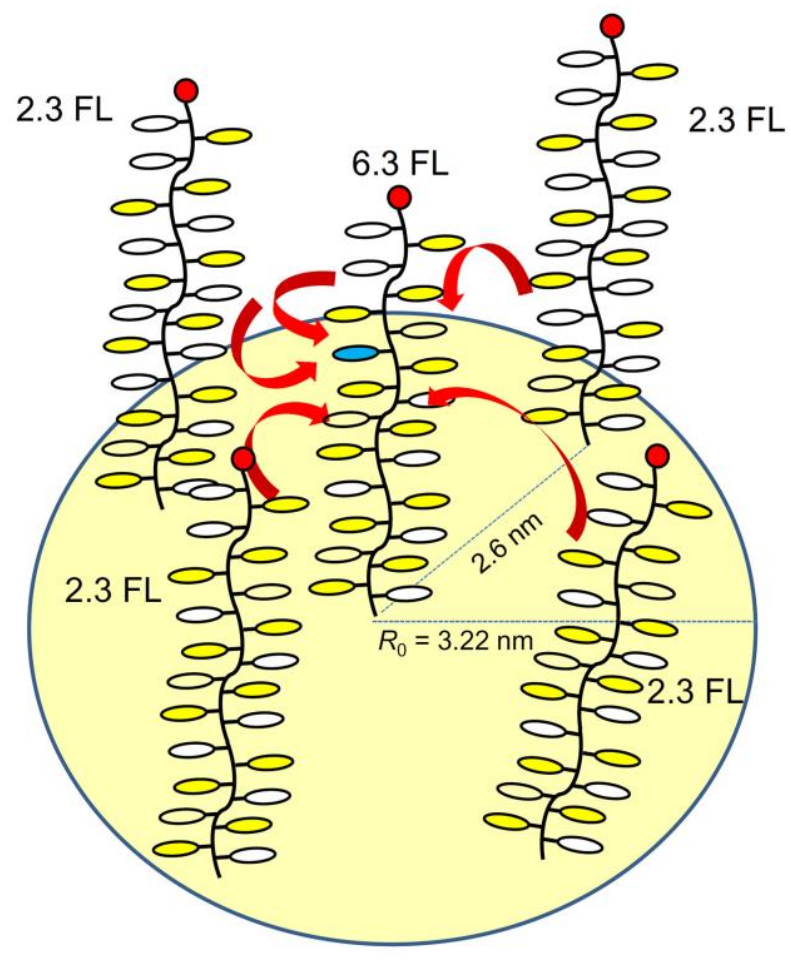

Figure 9. Förster distance and the number of fluorescence quenching.

\section{Conclusions}

We have synthesized DE-FL, poly(DE-co-FL), and SNP-poly(DE-co-FL) and investigated the fluorescence switching behavior. Fluorescence intensity of DE-FL decreased linearly with increasing photocyclization conversion. In contrast, fluorescence intensity of poly(DE-co-FL) and SNP-poly(DE-co-FL) significantly decreased with increasing photocyclization conversion. In particular, the number of fluorescence quenching per one DEc in SNP-poly(DE-co-FL) was much higher than that in DE-FL and poly(DE-co-FL). We succeeded in preparing a novel photochromic silica nanoparticle that exhibits efficient fluorescence switching.

\section{Supporting Information Summary}




\section{WILEY-VCH}

Supporting Information is available from the Wiley Online Library. It includes the detail experimental section, ${ }^{1} \mathrm{H}-\mathrm{NMR}$ spectrum of poly(DE-co-FL), illustration for coverage density of copolymer on the surface of SNP, absorption and fluorescence spectra, optical properties, and parameters for energy transfer.

\section{Acknowledgements}

This work was partly supported by JSPS KAKENHI Grant Number JP26107013 in Scientific Research on Innovative Areas "Photosynergetics". K.S. acknowledges Program for Leading Graduate Schools for System-inspired Leaders in Material Science: SiMS, Osaka Prefecture University/Osaka City University from MEXT, Japan. The authors also thank Osaka Gas Chemical Co., Ltd. and Nippon Zeon Co., Ltd. for providing chemicals.

\section{Conflict of Interest}

The authors declare no conflict of interest.

\section{Keywords:}

Diarylethene, Energy Transfer, Fluorescence Switching, Photochromism, Silica Nanoparticle

\section{References}

[1] G. H. Brown, Photochromsim, Wiley-Interscience, New York 1971.

[2] H. Dürr, H. Bouas-Laurent, Photochromism: molecules and systems, Elsevier, Amsterdam 2003.

[3] M. Irie, Chem. Rev. 2000, 100, 1685.

[4] S. Kobatake, M. Irie, Bull. Chem. Soc. Jpn. 2004, 77, 195.

[5] A. J. Myles, N. R. Branda, Adv. Funct. Mater. 2002, 12, 167.

[6] H. Tian, S. Wang, Chem. Commun. 2007, 781.

[7] E. Betzig, G. H. Patterson, R. Sougrat, O. W. Lindwasser, S. Olenych, J. S. Bonifacino, M. W. Davidson, J. Lippincott-Schwartz, H. F. Hess, Science 2006, 313, 1642.

[8] S. T. Hess, T. P. K. Girirajan, M. D. Mason, J. Biophys. 2006, 91, 4258.

[9] M. Irie, T. Fukaminato, K. Matsuda, S. Kobatake, Chem. Rev. 2014, 114, 12174.

[10] J. Su, T. Fukaminato, J. Placial, T. Onodera, R. Suzuki, H. Oikawa, A. Brosseau, F.

Brisset, R. Pansu, K. Nakatani, R. Métivier, Angew. Chem. Int. Ed. 2016, 128, 3726.

[11] M. Irie, T. Fukaminato, T. Sasaki, N. Tamai, T. Kawai, Nature 2002, 420, 759.

[12] T. Förster, Naturwiss 1946, 33, 166.

[13] T. Förster, Ann. Physik. 1948, 437, 55. 


\section{WILEY-VCH}

[14] Z. Zhou, H. Hu, H. Yang, T. Yi, K. Huang, M. Yu, F. Li, C. Huang, Chem. Commun. 2008, 39, 4786 .

[15] C. Li, H. Yan, L.-X. Zhao, G.-F. Zhang, Z. Hu, Z.-L. Huang, M.-Q. Zhu, Nature Commun. 2014, 5, 5709.

[16] H. Cho, E. Kin, Macromolecules 2002, 35, 8684.

[17] C. Li, W.-L. Gong, Z. Hu, M. P. Aldred, G.-F. Zhang, T. Chen, Z.-L. Huang, M.-Q. Zhu, RSC Adv. 2013, 3, 8967.

[18] K. Uno, H. Niikura, M. Morimoto, Y. Ishibashi, H. Miyasaka, M. Irie, J. Am. Chem. Soc. 2011, 133, 13558.

[19] J. Fölling, S. Polyakova, V. Belov, A. van Blaaderen, M. L. Bossi, S. W. Hell, Small 2008, 4,134 .

[20] R. Seno, S. Kobatake, Dyes Pigm. 2015, 114, 166.

[21] M. Ejaz, S. Yamamoto, K. Ohno, Y. Tsujii, T. Fukuda, Macromolecules 1998, 31, 5934.

[22] Y. Tsujii, M. Ejaz, K. Sato, A. Goto, T. Fukuda, Macromolecules 2001, 34, 8872.

[23] W. Stöber, A. Fink, E. Bohn, J. Colloid Interf. Sci. 1968, 26, 62.

[24] M. Ejaz, S. Yamamoto, K. Ohno, Y. Tsujii, T. Fukuda, Macromolecules 1998, 31, 5934.

[25] K. Ohno, T. Morinaga, K. Koh, Y. Tsujii, T. Fukuda, Macromolecules 2005, 38, 2137.

[26] A. V. Blaaderen, A. Vrij, J., Colloid Interface Sci. 1993, 156, 1.

[27] S. T. Milner, Science 1991, 251, 905. 


\section{WILEY-VCH}

\section{Table of Contents}

\section{Synthesis and Optical Properties of Fluorescent Switchable Silica Nanoparticles}

Covered with Copolymers Consisting of Diarylethene and Fluorene Derivatives

Katsuya Shimizu and Prof. Seiya Kobatake*



Fluorescence ON/OFF switching behavior of silica nanoparticles covered with polymers consisting of a photoswitchable diarylethene and a fluorescent fluorene was investigated. Fluorescence of the nanoparticle significantly decreased with increasing photocyclization conversion of the diarylethene. The diarylethene closed-ring forms quenched the fluorescence of the fluorene moieties not only in the same polymer chain but also in four polymer chains in the neighborhood. 